\title{
The Primordial Principle of Self-Interaction
}

\author{
Edwin Eugene Klingman (1) \\ Cybernetic Micro Systems, Inc., San Gregorio, CA, USA \\ Email: klingman@geneman.com
}

How to cite this paper: Klingman, E.E. (2021) The Primordial Principle of SelfInteraction. Journal of Modern Physics, 12, 65-81.

https://doi.org/10.4236/jmp.2021.122007

Received: December 4, 2020

Accepted: January 11, 2021

Published: January 14, 2021

Copyright $\odot 2021$ by author(s) and Scientific Research Publishing Inc. This work is licensed under the Creative Commons Attribution International License (CC BY 4.0).

http://creativecommons.org/licenses/by/4.0/

\begin{abstract}
The Standard Model of Particle Physics treats four fields-the gravitational, electromagnetic, weak and strong fields. These fields are assumed to converge to a single field at the big bang, but the theory has failed to produce this convergence. Our theory proposes one primordial field and analyzes the evolution of this field. The key assumption is that only the primordial field exists-if any change is to occur, it must be based upon self-interaction, as there is nothing other than the field itself to interact with. This can be formalized as the Principle of Self-interaction and the consequences explored. I show that this leads to the linearized Einstein field equations and discuss the key ontological implications of the theory.
\end{abstract}

\section{Keywords}

Self-Interaction, Principles of Physics, Electromagnetism, Gravitomagnetism, "Weak Field" Approximation, Kasner Metric, Iterated Solutions, Gauge Theory of Gravity, Primordial Principle

\section{Introduction}

Newton, in his analysis of gravity, concluded that it made no sense to imagine a truly empty vacuum with nothing in it; something must be there, to transmit force from one place to another. This "something" is the gravitational field. Later Einstein reached the same conclusion [1]: "there is no such thing as an empty space, i.e., a space without a field. Spacetime does not claim existence on its own, but only as a structural quality of the field... there exists no space 'empty of field". Einstein thus conceived of physical reality as a field.

The concept of field evolved from Faraday to Maxwell to Hertz [2]; then Heaviside [3] extended Newton's gravitation field in analogy with the electromagnetic field. Einstein's nonlinear gravity can be linearized to produce Heaviside's equations and the gravitational waves implied by these equations have been de- 
tected [4]. Contributors to quantum field theory assigned a gauge theory to every fundamental particle such that Feynman incorporated gravity as the $31^{\text {st }}$ field [5]. Eventually, Susskind [6] claimed the equivalent of up to 500 fields account for the multiverse.

The current status of gravitational field theory is marked by two questions. The Standard Model poses that electromagnetic, gravitomagnetic, weak and strong fields converge at the big bang, yet this convergence fails without supersymmetry, which has effectively been eliminated at the LHC [7]. This suggests new approaches be explored. The other question, posed by Will and Poisson [8], concerns the "unreasonable effectiveness" of the weak field approximation. This paper directly addresses this question.

The plan of this paper is as follows:

Section 1, the Introduction, traces the history of fields in physics and discusses the failure of convergence to a primordial field.

Section 2, the Principle of Self-interaction, introduces the concept of primordial field as the original entity that existed or came into existence "in the beginning". As nothing else existed, any physical interaction could only be with the primordial field itself. Based on this formulation of physics, I propose a "change operator", acting on the field and represent the change as the field acting on itself. This yields the self-interaction equation. I then solve this equation for a scalar aspect of the field and interpret this scalar as time, in which case the solution has a frequency property. I then consider a vector field aspect and formulate a vector equation.

Section 3 treats "contact with Newton's equation" as a means of linking the symbolic formulation of self-interaction to physical reality. We find that Newton's equation of gravity can be derived from the self-interaction equation, and compare the primordial self-interaction to the interaction between separate entities.

Section 4 introduces Hestenes' Geometric Calculus as the most appropriate mathematical formalism for physics, based on the fact that every geometric algebra entity has both an algebraic interpretation and a geometric interpretation. In addition, Geometric Calculus deals with multi-vectors composed of different types of entities. For example, we found a scalar solution and a vector solution to the self-interaction equation; therefore we combine both solutions into a multi-vector representation. The geometric product of two vectors is introduced as the fundamental operation and the dual operator, $i$, is also introduced. The self-interaction equation is redefined in the Geometric Calculus formalism.

Section 5 expands the redefined self-interaction equation in terms of the fundamental constituents introduced in section 4 , and then the expansion of the equation is regrouped in terms of "like terms" appearing on each side of the equation. This process yields four equations which are presented in terms of the fundamental constituents.

Section 6 interprets the four equations, derived in section 5, in terms of the associated physics; the result is a set of equations known as the "weak field equa- 
tions" of general relativity.

Section 7 derives the gravitomagnetic wave equations and discusses the recent detection of gravitomagnetic waves.

Section 8 discusses momentum in the primordial field and describes how terms that would seem to cancel mathematically can exist if separated in space. The addition of these terms completes the linearized field equations first derived by Heaviside and later derived from Einstein's general relativistic nonlinear field equations.

Section 9 discusses the fact that it has been impossible to successfully apply adjunct linear "Lorenz condition" stipulation to Einstein's "generally covariant" formulation of general relativity. The Geometric Calculus is used to formulate the gravitomagnetic gauge field equations and to derive the Lorenz gauge condition.

Section 10 presents the key finding of the theory of gravity based on the Principle of Self-interaction: the fact that "field strength" does not appear in this theory. That distinguishes our linear gravitomagnetic field equations from the equivalent field equations derived from general relativity. Relativists assume that these equations apply only for "weak" gravitational fields, whereas our theory is "strength-independent", and applies for all strengths.

Section 11 discusses the fact that Einstein's nonlinear field equations can be derived iteratively from the linearized field equations we have derived from our theory of the self-interacting primordial field.

Section 12 presents conclusions and reiterates the "strength-independent" nature of the self-interaction solution, enabling a physical interpretation of the Kasner metric solution of Einstein's equations. Other applications will be treated in future papers. We summarize by emphasizing that self-interaction theory of gravity presents a novel reinterpretation of gravity that addresses current confusions associated with the success of the post-Newtonian approach to relativity.

\section{The Principle of Self-Interaction}

Perhaps the simplest assumption upon which to base a universe is that the universe either existed, or came into existence, as a primordial entity. That is, "in the beginning" this primordial entity, and nothing else, existed. If nothing else existed, there was nothing to interact with the primordial entity except itself. To be specific we call this entity a physical field.

Our physics tools are generally designed to relate changes in one physical entity to another entity. For example the basic equation

$$
\nabla f=s
$$

relates change (represented by operator $\nabla$, undefined) in a field (represented by $f$, undefined) to a source $s$. If our primordial field is represented by $f$, and change is represented by mathematical operator $\nabla$, then $s$ does not exist apart from $f$. And change must have occurred if the primordial field evolved to the current state of our universe. Today, changes occur when things "interact with each other", but, if nothing else existed to interact with the primordial field, any 
interaction could only be the field interacting with itself, and we denote this by ff. So change based on self-interaction is described by the equation

$$
\nabla f=f f
$$

This fundamental Principle of Self-interaction describes changes in the primordial field $f$ as it interacts with itself.

In the following, we assume minimal knowledge of physics, while at the same time we assume knowledge of logic and mathematics, as necessary. As we cannot model the universe based only on two symbols $\nabla$ and $f$, we assume some aspect or property $p$ of the field and ask how the field changes with respect to this aspect. In other words $\nabla=\partial / \partial p$ where $f=f(p)$. The self-interaction equation becomes:

$$
\frac{\partial}{\partial p} f(p)=f(p) f(p) .
$$

This fundamental equation has solution, $f=-p^{-1}$.

$$
\frac{\partial}{\partial p}\left(-p^{-1}\right)=\left(-p^{-1}\right)\left(-p^{-1}\right) \Rightarrow-\left(-p^{-2}\right)=+p^{-2}
$$

This implies a scalar property or aspect of the primordial universe. The most fundamental scalar in physics is almost certainly time $t$, therefore we initially identify $p=t$ and $f(t)=-1 / t$. The field appears to have a frequency aspect and we postpone interpretation of the - sign. Parameter $t$ is cosmological time, the same time everywhere in the universe. It represents a distance (duration in time) from a beginning to the present state. To find a 3-space vector solution to $\nabla f=f f$ we promote $\nabla$ and $f$ to $\vec{\nabla}$ and $\vec{f}$ for parameter $\vec{r}=\{x, y, z\}$. The self-interaction equation becomes

$$
\vec{\nabla} \vec{f}=\overrightarrow{f f} .
$$

This combination is not well defined in vector calculus, so we expand the meaning of the change operation to project change onto the field, and use the inner product or dot product to represent self-interaction:

$$
\vec{\nabla} \cdot \vec{f}=\vec{f} \cdot \vec{f}
$$

Physical fields have energy density proportional to the square of the fields; $\rho_{E}=\vec{f} \cdot \vec{f}=f^{2}$. For unity speed of light $(c=1)$ we have mass equivalent density $\rho_{m}=\rho_{E}$ and we find $\vec{\nabla} \cdot \vec{f}=\rho_{E}$.

\section{Contact with Newton's Gravity}

Einstein's general relativity field equations are unphysical differential geometry equations unless, and until, they make contact with Newton's equation; we apply the same criterion to the primordial field equation. Specifically, we write

$$
\vec{\nabla} \cdot \vec{G}=\vec{G} \cdot \vec{G} .
$$

If we assume that $\vec{f}$ is gravitational field $\vec{G}$, we recall that, unlike the electromagnetic field, the gravitational field energy is negative, as it is necessary to 
add energy to free a body captured in a gravity well. Thus energy density $\rho_{E}$ is negative and (for $c=1$ ) $\rho_{m}=\rho_{E}$ and the self-interaction equation becomes

$$
\vec{\nabla} \cdot \vec{G}=-\rho_{m} .
$$

If Newton's gravitational constant $g=1$, this is seen to be Newton's equation of the gravitational field. Therefore our primordial field is tentatively identified as the gravitational field. Although it may be assumed that gravity is sourced by mass, Calabi [9] asked: "Could there be gravity in our universe even if space is vacuum totally devoid of matter?" His answer, that curvature makes gravity without matter possible, establishes a feasible identification of the primordial field. Let us return to the self-interaction Equation (7). Danforth first showed, [10] circa 2007, that this equation has solution

$$
\vec{G}(\vec{r})=\frac{1}{\vec{r}} \equiv \frac{\vec{r}}{r^{2}} \text { such that } \vec{G} \cdot \vec{G}=\frac{1}{r^{2}}=\rho_{E}
$$

We compare this with Newton's force law

$$
\vec{F}=-\frac{g m m^{\prime}}{r^{2}}=-g m m^{\prime} \frac{\vec{r}}{r^{3}} \equiv m^{\prime} \vec{G}_{N}
$$

describing the force of gravity on test mass $m^{\prime}$ a distance $r$ from the source of the field, $m$. Let us use unit test mass $m^{\prime}=1$ and keep $g=1$ and set $\vec{G}_{N}=\vec{F} / m^{\prime}=\left(m / r^{3}\right) \vec{r}=\rho_{m} \vec{r}$. Although we have suppressed the display of $m^{\prime}$ and $g$, these constants enter into any dimensional check, so that if Newton's gravitational constant has units $|g|=l^{3} / m t^{2}$ we find $\left|\vec{G}_{N}\right|=|g \rho r|=\left|\frac{l^{3}}{m t^{2}} \frac{m}{l^{3}} \frac{l}{1}\right|=\frac{l}{t^{2}}$, which correctly has dimensions of acceleration.

In the following, we set Newton's gravitational constant $g=1$. This scalar dimensional constant is always present and has dimensions $|g|=l^{3} / m t^{2}$ whether we display it or suppress its display. Similarly, we let test mass $m^{\prime}=1$ retaining the result shown in Equation (10): $\vec{F}=m^{\prime} \vec{G}_{N}$. Thus:

$$
\begin{aligned}
& \text { Newtonian: } \vec{G}_{N}=\frac{g m \vec{r}}{r^{3}} \\
& \text { Primordial: } \vec{G}=\frac{g \vec{r}}{r^{2}}
\end{aligned}
$$

If the forces are identical these two equations imply $m=r$. We choose the origin of the gravitational system $(0,0,0)$ to be the center-of-mass and consider the test mass $m^{\prime}$ to be located at $\vec{r}=(x, y, z) \equiv(r, \theta, \phi)$. In Newtonian problems, the mass $m$ is located at the origin and $m^{\prime}$ at $\vec{r}$.

We see that the gravitational field derived from Newton's force law has dependence $\vec{G}_{N} \sim \vec{r} / r^{3}$ while the solution of the primordial field self-interaction Equation (9) has dependence $\vec{G} \sim \vec{r} / r^{2}$. How can we explain this difference?

The difference between the gravitational acceleration due to fixed mass $m$, distance $r$ from the test mass $m^{\prime}$, and a primordial field $\vec{G}$ tested at the same point (see Figure 1 ) is as follows: for mass $m$ all of the mass contributes to the 


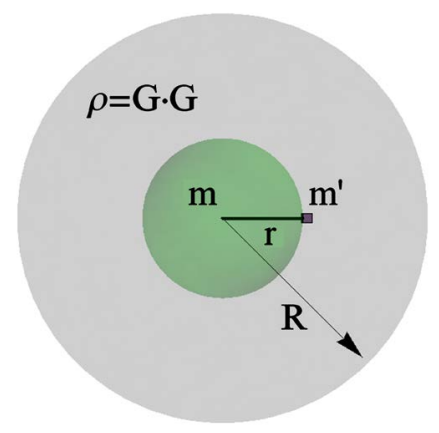

Figure 1. Mass $m$ at $\vec{r}$.

field $\vec{G}$ at $\vec{r}$. For the primordial field, assumed spherically symmetric, Birkhoff's Shell theorem [11] implies that only the mass density inside the sphere of radius $r$ (centered on the origin at the "center of mass") contributes to the field at the test point. Mass $m$ inside the sphere is (modulo $4 \pi / 3$ )

$$
m=r^{3}(\rho)=r^{3}(\vec{G} \cdot \vec{G})=r^{3}\left(\frac{1}{r^{2}}\right)=r .
$$

This is the result implied by Equations (11) and (12). Thus the Newtonian force on the test mass at $\vec{r}$, due to mass $m$ inside the sphere, is

$$
\vec{G}_{N}=\frac{\vec{F}}{m^{\prime}}=\frac{g m \vec{r}}{r^{3}} \Rightarrow \frac{g r \vec{r}}{r^{3}}=\frac{g \vec{r}}{r^{2}}=G(r),
$$

and self-interaction of primordial field at $\vec{r}$ is correctly given by the self-interaction equation. In order to derive this result, we modified equation $\vec{\nabla} \vec{f}=\overrightarrow{f f}$ by specializing in the inner product represented by “.". We next generalize our tool-set to solve the unmodified equation.

\section{Geometric Calculus}

Our goal is to create a physical model, or a physical theory of reality, based on minimal knowledge of physics at the time of creation and on the most effective mathematics. As our world has both logical relationships and shapes, the only mathematical field in which every mathematical term has both an algebraic and a geometric interpretation is Hestenes' geometric calculus [12], with its fundamental theorem on a smooth $m$-dimensional manifold $M$ with boundary $\partial M$ :

$$
\int_{M} \mathrm{~d}^{m} x \partial F=\oint_{\partial m} \mathrm{~d}^{m-1} x F
$$

This theorem is compatible with and contains Gauss's theorem, Stokes theorem, Green's theorem, and the Cauchy integral formula, in coordinate-free formalism [13]. The type of geometric algebra entities in a $(3+1) \mathrm{D}$ universe are scalar, vector, bivector, and trivector or pseudo-scalar. The fundamental geometric algebra operation, the geometric product of two vectors $\vec{u}$ and $\vec{v}$, is:

$$
\vec{u} \vec{v}=\vec{u} \cdot \vec{v}+\vec{u} \wedge \vec{v} .
$$

The geometric product of two vectors yields a multi-vector consisting of inner product $\vec{u} \cdot \vec{v}$, which is a scalar projecting one vector into the other, and outer 
product $\vec{u} \wedge \vec{v}$, which is a bivector, a directed area representing the rotation of $\vec{u}$ into $\vec{v}$. The scalar product is identical to the vector dot product. The bivector can be related to the vector cross product as seen in Figure 2.

$$
\vec{u} \wedge \vec{v}=i \vec{u} \times \vec{v}
$$

Bivector $\vec{u} \wedge \vec{v}$ is a directed area with no defined shape, while $\vec{u} \times \vec{v}$ is an axial vector, which is not included as a vector in vector analysis, but which is required to represent magnetic fields. The term $i$ is the dual operator that transforms cross-product into wedge, as shown, or vice versa: $\vec{u} \times \vec{v}=-i \vec{u} \wedge \vec{v}$. An axial vector can be envisioned as a vector cross product, but its rotational aspect can be represented as a bivector. The negative sign associated with $-t^{-1}$ is interpreted to mean left-handed circulation of the local field with rotational frequency $\sim t^{-1}$.

In geometric calculus, as in vector calculus, the derivative operator $\vec{\nabla}$ is viewed as a vector. Therefore the geometric product of $\vec{\nabla}$ with field $\vec{f}$ is as follows

$$
\vec{\nabla} \vec{f}=\vec{\nabla} \cdot \vec{f}+\vec{\nabla} \wedge \vec{f} . \quad \text { gradient }=\text { divergence }+ \text { curl }
$$

This relation gradient $=$ divergence + curl is not true in any other mathematical formalism.

Scalar derivative $\partial_{t}$ operating on scalar function $f(t)$ yields $f(t)=-t^{-1}$ for self-interaction equation $\partial_{t} f(t)=f(t) f(t)$, while a primordial field with aspects of distance in time and space leads to a directional field $\vec{f}$. If these aspects are separable with respect to time and space, we resolve our field into two primary subfields, $\vec{G}(r)$ and $\vec{C}(t)$ and express the primordial field

$$
\bar{f}=\vec{G}+i \vec{C}
$$

where $\vec{G}(r)$ is a vector and $\vec{C}(t)$ is a bivector formed by the dual operator $i$ operating on the $\vec{C}(t)$ field vector. The nature of $\vec{G} \sim \vec{r}^{-1}$ and the nature of $\vec{C} \sim-t^{-1}$, where the $\sim$ symbol implies proportionality; scalar constants may be required to match experimental measurement of $\vec{G}(r)$ and $\vec{C}(t)$. The overbar denotes a multivector. If $\vec{\nabla}$ is the vector derivative with respect to space, and $\partial_{t}$ is the scalar derivative with respect to time, then for changes in space and time, we generalize change operator $\bar{\nabla}$ to include both derivatives, and field $\bar{f}$ to include both subfields:

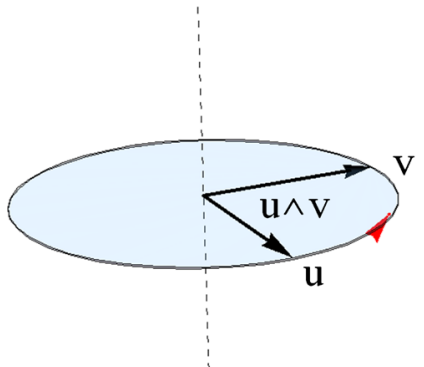

(a)

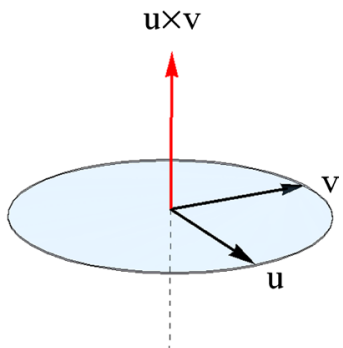

(b)

Figure 2. (a) Wedge product; (b) Cross product. 


$$
\bar{\nabla} \bar{f}=\overline{f f} \Rightarrow\left(\vec{\nabla}+\partial_{t}\right)(\vec{G}+i \vec{C})=(\vec{G}+i \vec{C})(\vec{G}+i \vec{C})
$$

\section{Expansion of Primordial Field Equation}

$$
\left(\vec{\nabla}+\partial_{t}\right)(\vec{G}+i \vec{C})=(\vec{G}+i \vec{C})(\vec{G}+i \vec{C})
$$

First we multiply out all terms, noting that the dual operator $i$ commutes with all vectors.

$$
\vec{\nabla} \vec{G}+\partial_{t} \vec{G}+i \vec{\nabla} \vec{C}+i \partial_{t} \vec{C}=\vec{G} \vec{G}+i \vec{G} \vec{C}+i \vec{C} \vec{G}-\vec{C} \vec{C}
$$

Next we expand the geometric products on both sides and then group like terms.

$$
\begin{aligned}
& \vec{\nabla} \cdot \vec{G}+i \vec{\nabla} \times \vec{G}+\partial_{t} \vec{G}+i \vec{\nabla} \cdot \vec{C}-\vec{\nabla} \times \vec{C}+i \partial_{t} \vec{C} \\
& =\vec{G} \cdot \vec{G}+i \vec{G} \times \vec{G}+i \vec{G} \cdot \vec{C}-\vec{G} \times \vec{C}+i \vec{C} \cdot \vec{G}-\vec{C} \times \vec{G}-\vec{C} \cdot \vec{C}-i \vec{C} \times \vec{C}
\end{aligned}
$$

Observing that a curl of a vector with itself is identically zero we delete terms $\vec{G} \times \vec{G}$ and $\vec{C} \times \vec{C}$. We also note that $\vec{G} \times \vec{C}+\vec{C} \times \vec{G}=0$. The remaining terms should be grouped by like terms.

$$
\vec{\nabla} \cdot \vec{G}+i \vec{\nabla} \times \vec{G}+\partial_{t} \vec{G}+i \vec{\nabla} \cdot \vec{C}-\vec{\nabla} \times \vec{C}+i \partial_{t} \vec{C}=\vec{G} \cdot \vec{G}+i \vec{G} \cdot \vec{C}+i \vec{C} \cdot \vec{G}-\vec{C} \cdot \vec{C}
$$

First we group scalars; next scalars multiplied by the dual operator, then vector terms and finally vectors multiplied by $i$. This expansion of the self-interaction equation yields four equations:

$$
\begin{gathered}
\vec{\nabla} \cdot \vec{G}=\vec{G} \cdot \vec{G}-\vec{C} \cdot \vec{C} \\
i \vec{\nabla} \cdot \vec{C}=i 2 \vec{G} \cdot \vec{C} \\
\partial_{t} \vec{G}-\vec{\nabla} \times \vec{C}=0 \\
i \vec{\nabla} \times \vec{G}+i \partial_{t} \vec{C}=0
\end{gathered}
$$

These equations derive from the self-interaction of the primordial field according to our Self-interaction Principle, based on the simplest assumptions. They are quite explicit, yet to proceed further we need to make use of what more we know of physical reality. For example, we know that physical fields are real and have energy. Ohanian and Ruffini state: [14] "The gravitational field may be regarded as the material medium sought by Newton; the field is material because it possesses an energy density." For example, energy-momentum density of the electromagnetic fields $\vec{E}$ and $\vec{B}$ are given by $E^{2}+B^{2}+\vec{E} \times \vec{B}$. Therefore we assume that $\vec{G} \cdot \vec{G}$ and $\vec{C} \cdot \vec{C}$ represent energy density, and $\vec{G} \times \vec{C}$ represents momentum density. We know that energy has mass equivalence such that energy density $\rho_{E}=\rho_{m} c^{2}$; if $c=1$ then $\rho_{E}=\rho_{m}$. Additionally, we know that Einstein's general relativity field equations are simply differential geometry until they make contact with real physics in the form of Newton's equation. Therefore we conclude that we too must again make contact with Newton's equation.

\section{Interpretation of Primordial Field Equation}

When we apply our knowledge to the first scalar Equation (22a), we interpret 
$\vec{G}$ as the gravitational field and $\vec{G} \cdot \vec{G}$ as the self-energy density of the gravitational field. Ignoring the complication of $\vec{C} \cdot \vec{C}$ we have $\vec{\nabla} \cdot \vec{G}=\rho$. Calling on additional information, we know that gravitational energy is negative since we must apply positive energy to a body captured in a gravitational field in order for it to escape the field, hence the mass density $\rho \sim-\vec{G} \cdot \vec{G}$ and we obtain:

$$
\vec{\nabla} \cdot \vec{G}=-\rho \text { Newton's gravitational equation }
$$

This implies that $\vec{C} \cdot \vec{C}$ has positive energy so that equivalent mass density $-\vec{C} \cdot \vec{C}$ in Equation (22a) contributes correctly to Newton's equation. It has recently been shown that rotational energy in molecules is equivalent to mass [15], therefore the circulational energy of the C-field yields the appropriate sign. In other words, we have derived Newton's equation of the gravitational field from our Principle of Self-interaction as required.

Our next interpretation also relies on analogy with the electromagnetic field, where $\vec{E} \cdot \vec{B}=0$, since the fields are orthogonal to each other. Obviously, our $\mathrm{C}$-field is the gravitomagnetic field, sometimes called cogravitation [16]. Existence of this field was positively established circa 2011 by the Gravity Probe B experiment [17]. If we assume that $\vec{G} \cdot \vec{C}=0$ then Equation (22b) becomes

$$
\vec{\nabla} \cdot \vec{C}=0 \text {. }
$$

Again analogous to $\vec{\nabla} \cdot \vec{B}=0$, this implies that no gravitomagnetic "pole" exists and also that the gravitomagnetic field can be derived from a gauge equation $\vec{C}=\vec{\nabla} \times \vec{A}$ since $\vec{\nabla} \cdot \vec{\nabla} \times \bar{A} \equiv 0$.

We obtained terms $-\vec{C} \times \vec{G}$ and $-\vec{G} \times \vec{C}$. When we add these and note that $\vec{G} \times \vec{C}=-\vec{C} \times \vec{G}$ we initially assume that these terms cancel. We will revisit these terms later. Until then we have:

$$
\vec{\nabla} \times \vec{C}=\partial_{t} \vec{G}
$$

By now it's obvious from the electromagnetic analogy that the last equation is

$$
\vec{\nabla} \times \vec{G}=-\partial_{t} \vec{C}
$$

Grouping these for convenience we obtain:

$$
\begin{array}{ll}
\vec{\nabla} \cdot \vec{G}=-\rho, & \vec{\nabla} \times \vec{G}=-\partial_{t} \vec{C} \\
\vec{\nabla} \cdot \vec{C}=0, & \vec{\nabla} \times \vec{C}=+\partial_{t} \vec{G}
\end{array}
$$

These equations, derived from the Self-interaction Principle, were derived by Oliver Heaviside in 1893, and later from Einstein's relativistic field equations, as the "weak field equations".

\section{Gravitational Wave Equations}

The Maxwell-like field equations invite the following procedure, based on the vector identity:

$$
\vec{\nabla} \times(\vec{\nabla} \times \vec{V})=\vec{\nabla}(\vec{\nabla} \cdot \vec{V})-\nabla^{2} \vec{V},
$$

where the last term can be written $-\vec{\nabla} \cdot(\vec{\nabla} \vec{V})$. The first term on the right va- 
nishes at all times for $\vec{V}=\vec{C}$ since $\vec{\nabla} \cdot \vec{C} \equiv 0$. For no mass density $\rho=0$, and for minimal field density $\rho \approx 0$, we have $\vec{\nabla} \cdot \vec{G} \approx 0$, leaving the relation $\vec{\nabla} \times(\vec{\nabla} \times \vec{V})=-\nabla^{2} \vec{V}$. Substitute first $\vec{G}$ and then $\vec{C}$ into this identity.

$$
\begin{aligned}
& \vec{\nabla} \times(\vec{\nabla} \times \vec{G})=-\nabla^{2} \vec{G} \Rightarrow \vec{\nabla} \times\left(-\frac{\partial \vec{C}}{\partial t}\right)=-\frac{\partial}{\partial t}(\vec{\nabla} \times \vec{C}) \Rightarrow-\frac{\partial^{2} \vec{G}}{\partial t^{2}} \\
& \vec{\nabla} \times(\vec{\nabla} \times \vec{C})=-\nabla^{2} \vec{C} \Rightarrow \vec{\nabla} \times\left(+\frac{\partial \vec{G}}{\partial t}\right)=+\frac{\partial}{\partial t}(\vec{\nabla} \times \vec{G}) \Rightarrow-\frac{\partial^{2} \vec{C}}{\partial t^{2}}
\end{aligned}
$$

Summarizing, we have obtained the wave equations

$$
-\nabla^{2} \vec{G}+\frac{\partial^{2} \vec{G}}{\partial t^{2}}=0 \text { and }-\nabla^{2} \vec{C}+\frac{\partial^{2} \vec{C}}{\partial t^{2}}=0
$$

Dimensional analysis indicates that a velocity-squared term is needed, so we assume $v=1$ and include the symbolic speed in the equation.

$$
-\nabla^{2} \vec{G}+\frac{1}{v^{2}} \frac{\partial^{2} \vec{G}}{\partial t^{2}}=0, \quad-\nabla^{2} \vec{C}+\frac{1}{v^{2}} \frac{\partial^{2} \vec{C}}{\partial t^{2}}=0
$$

The 2017 [18] detection of inspiralling neutron stars established that the speed of light in an absolute frame, defined by the Cosmic Microwave Background, is the same as the speed of propagation of gravity through the same frame which is pervaded by gravity. Will [19] analyzes the connection between gravity and speed of light by correlating electromagnetic parameters $\mu, \varepsilon$ with Newton's gravitational constant $g$ in terms of $T H \varepsilon \mu$ formalism of Lightman and Lee. We have:

$$
c=\frac{1}{\sqrt{\varepsilon_{0} \mu_{0}}} \Rightarrow[\varepsilon(g) \mu(g)]^{-1 / 2}=\left[\left(\frac{-1}{4 \pi g}\right)_{\varepsilon}\left(\frac{-4 \pi g}{c^{2}}\right)_{\mu}\right]^{-1 / 2}=\left(c^{-2}\right)^{-1 / 2}=c
$$

Since it is now known that velocity $v$ in Equations (28) is equal to the speed of light we observe that the Principle of Self-interaction predicts gravitational waves.

\section{Momentum in the Primordial Field}

We now revisit the two terms appearing in the expansion of the Self-interaction Equation (21c) which we deleted based on $\vec{G} \times \vec{C}+\vec{C} \times \vec{G}=0$. We did so due to antisymmetry $\vec{G} \times \vec{C}=-\vec{C} \times \vec{G}$. However since $\vec{E} \times \vec{B}$ is the momentum energy density of the electromagnetic field; we interpret $-\vec{C} \times \vec{G}$ as the momentum energy density of the gravitomagnetic field. The energy density has equivalent mass density, and momentum density implies that energy is moving with velocity $\vec{v}$. Hence we rewrite $-\vec{C} \times \vec{G}$ as $-\rho \vec{v}$ and obtain the complete set of Heaviside equations:

$$
\begin{array}{ll}
\vec{\nabla} \cdot \vec{G}=-\rho, & \vec{\nabla} \times \vec{G}=-\partial_{t} \vec{C} \\
\vec{\nabla} \cdot \vec{C}=0, & \vec{\nabla} \times \vec{C}=-\rho \vec{v}+\partial_{t} \vec{G}
\end{array}
$$

This requires some physical explanation. First, we note that $\vec{G} \times \vec{C}$ cancels $\vec{C} \times \vec{G}$ if they are the same vectors. Based on the significance of the gravitational 


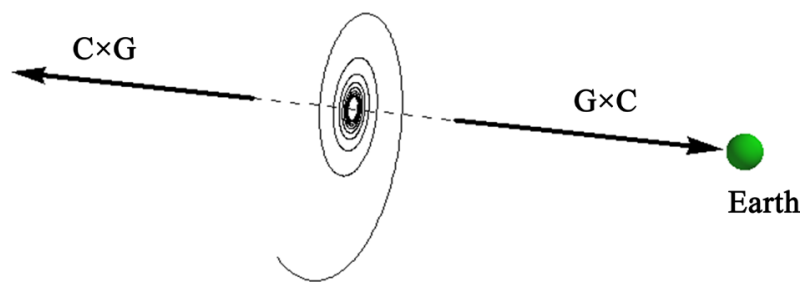

Figure 3. Inspiraling stars.

waves detected from in-spiraling neutron stars, we re-examine this interpretation. Obviously, observed from Earth, the momentum expressed by $\vec{G} \times \vec{C}$ is $\vec{G}(\vec{r}) \times \vec{C}(t)$ as symbolized in Figure 3.

But imagine that the earth is positioned at the far side of the inspiraling stars. In this case, the momentum vector observed would be $\vec{G}(-\vec{r}) \times \vec{C}(t)$ and would have the opposite apparent circulation, changing the sign of the momentum term. The position of Earth is arbitrary, and physics tells us that the inspiraling stars produced gravitational radiation in both directions, so we conclude that, instead of canceling, both momentum terms exist, and that is why we include the $\rho \vec{v}$ term in Equation (30). Finally, we generalized from the velocity $v=c$ of the gravitational radiation to encompass subluminal momentum density with $v<c$. Physical reasoning causes us to restore the terms that we originally canceled for mathematical reasons.

\section{Gauge Field Equations}

We anticipate problems with energy-momentum tensors in general relativity; unambiguous gauge fields simply cannot be defined, therefore we will reformulate our field Equation (21) by defining $\rho=\vec{G} \cdot \vec{G}-\vec{C} \cdot \vec{C}$ and $\vec{p}=\rho \vec{v} \sim \vec{G} \times \vec{C}$ where mass density current $\vec{p}$ (momentum density) is the analog of the electromagnetic charge density current $\vec{j}$. The source multivector becomes $\bar{p}=\rho+\vec{p} \equiv \rho(1+\vec{v})$ and the reformulated multivector field equation becomes:

$$
\left(\vec{\nabla}+\partial_{t}\right) \bar{f}=\bar{p} .
$$

As we have seen, this structure supports the wave operator:

$$
\partial_{\mu} \partial^{\mu}=\left(\nabla^{2}-\partial_{t}^{2}\right) \text {. }
$$

The following, modeled after Arthur [20] is motivated by Kauffmann's [21] statement that:

"There is no way to successfully apply adjunct linear "Lorenz condition" stipulation to the Einstein equation when it is presented in the customary "generally covariant" form:

$$
G_{\mu \nu}=-\left(8 \pi g / c^{2}\right) T_{\mu \nu} .
$$

Let us multiply Equation (31) by operator $\left(\vec{\nabla}-\partial_{t}\right)$ to obtain the sourceless wave equation on the left-hand side and the term $\left(\vec{\nabla}-\partial_{t}\right)(\rho+\vec{p})$ on the right, 
and recall that the scalar and vector potentials of electromagnetism give rise to scalar wave equations with $\rho$ and $\vec{j}$ as multivector source $\bar{j}=\rho+\vec{j}$. Arthur suggests that we find a single wave equation relating the four momentum $\bar{A}$ to $\bar{j}$. Our analogy replaces current density $\vec{j}$ by momentum density $\vec{p}$ to obtain

$$
\left(\nabla^{2}-\partial_{t}^{2}\right) \bar{A}=\bar{p}
$$

which, using Equation (31), we rewrite as

$$
\left(\vec{\nabla}+\partial_{t}\right)\left(\vec{\nabla}-\partial_{t}\right) \bar{A}=\left(\vec{\nabla}+\partial_{t}\right) \bar{f}
$$

This allows us to factor $\left(\vec{\nabla}+\partial_{t}\right)$ from each side to obtain

$$
\bar{f}=\left(\vec{\nabla}-\partial_{t}\right) \bar{A}+\bar{f}^{\prime}
$$

where $\bar{f}^{\prime}$ is any solution of the homogeneous (source free) Equation (28); $\left(\vec{\nabla}+\partial_{t}\right) \bar{f}^{\prime}=0$. For simplicity, we choose $\bar{f}^{\prime}=0$, and recall that $\left(\nabla^{2}-\partial_{t}^{2}\right)$ is a scalar. Therefore, if we multiply $\bar{A}$ by a scalar in Equation (34), we see that, since the multivector $\bar{p}$ is a scalar plus a vector, and $\bar{A}$ must have the same form as $\bar{p}$, we must write a multivector

$$
\bar{A}=-\phi+\vec{A}
$$

which is analogous to the electromagnetic gauge field four-vector. Next expand Equation (36):

$$
\begin{gathered}
\bar{f}=\left(\vec{\nabla}-\partial_{t}\right) \bar{A}=\left(\vec{\nabla}-\partial_{t}\right)(-\phi+\vec{A}) \\
\vec{G}+i \vec{C}=-\vec{\nabla} \phi+\vec{\nabla} \cdot \vec{A}+\vec{\nabla} \wedge \vec{A}+\partial_{t} \phi-\partial_{t} \vec{A}
\end{gathered}
$$

As always, each type of term must satisfy the equation separately; so, matching scalar, vector, and bivector terms, we obtain the following equations:

$$
\begin{gathered}
0=\partial_{t} \phi+\vec{\nabla} \cdot \vec{A} \\
\vec{G}=-\vec{\nabla} \phi-\partial_{t} \vec{A} \\
i \vec{C}=\vec{\nabla} \wedge \vec{A} \Leftrightarrow \vec{C}=\vec{\nabla} \times \vec{A}
\end{gathered}
$$

Since the field $\bar{f}$ has no scalar terms, we set the scalar terms to zero. From Newton's theory, we have $\phi \sim m / r$, thus the gravitational field agrees with Newton plus a gauge term. The last Equation (39c) follows from (23b) $\vec{\nabla} \cdot \vec{C}=0$. A dimensional analysis performed almost anywhere along the way will suggest that the gauge field $\vec{A}$ has dimensions of velocity $\vec{A} \sim \vec{v}$. In analogy with electromagnetic theory, the product of charge $q$ with gauge field $\vec{A}$ yields electromagnetic momentum $\vec{p}_{q}=q \vec{A}$, therefore our analogous product of mass with gauge field yields gravitomagnetic gauge field momentum $\vec{p}_{m}=m \vec{A} \equiv m \vec{v}$. Since this is the momentum in gravitomagnetism, we assume our assignment $\vec{A} \Rightarrow \vec{v}$ is correct. We check this by examining term $-\partial_{t} \vec{A}$ in Equation (39), where we find that the term represents an acceleration $\partial_{t} \vec{v}$ that is dimensionally compatible with gravitational acceleration $\vec{G}$. 
Finally, scalar Equation (39a) is dimensionally correct, since $\vec{\nabla} \cdot \vec{A} \sim \frac{\partial}{\partial x} \frac{\partial x}{\partial t} \sim \frac{\partial}{\partial t}$. But most significantly, this equation, in electromagnetic field theory is the Lorenz condition, and thus we consider this the gravitomagnetic Lorenz condition that is missing in general relativity. Great effort has been expended in relativity to establish an adjunct stipulation of the Lorenz condition $\partial_{\mu} A^{\mu}=0$. Kauffmann substitutes this into the "poster child" of gauge imposition in electromagnetic theory, $\partial_{\mu} \partial^{\mu} A^{v}-\partial^{v} \partial_{\mu} A^{\mu}=j^{v}$, and simplifies the relation to

$$
\partial_{\mu} \partial^{\mu} A^{v}=j^{v} \text {. }
$$

This "stipulation" is equivalent to Equation (34), suggesting that our approach has been correct. In summary, the much desired gravitomagnetic Lorenz gauge condition that is still missing from general relativity is obtained rather directly from the Principle of Self-interaction.

\section{Consequences of the Self-Interaction Principle}

The key factor concerning our derivation of the Heaviside-Einstein equations (30) is the fact that "field strength" never enters the equation. Our description of the field as "primordial" implies field strengths associated with the big bang, in strong contrast to the century old perception of weak field approximation. The Self-Interaction Principle replaces the "weak field approximation" with the "all-field equations"-the equations hold for all finite strengths of the gravitational field. That is the key lesson to be learned from this theory.

Will observes that "most of our understanding of gravitational radiation has come from approximations to Einstein's equations." And Padmanabhan analyzes a Lagrangian for the two body problem in the post-Newtonian approximation and finds that the perihelion precession per orbit "miraculously matches with the corresponding expression for a test body in the Schwarzschild metric. No simple reason for this conclusion is known and it is an issue worth thinking about".

The self-interaction theory contradicts this prevailing view by applying to any gravitational field, regardless of strength. It addresses Will's statement that "we have no good understanding of why this approximation to general relativity should be so effective." Will reviews binary pulsars and inspiralling compact binaries, including black holes, and the surprising fact that the approximate calculations agree with those of numerical relativity for very strong fields. He notes no obvious reason to expect weak field equations to work for inspiralling black holes, but they do.

The Self-Interaction Principle provides the reason. It is "strength-independent"; it never makes assumptions about "weak field" approximation.

\section{The Equivalence of Linear and Nonlinear Formulation of Gravitation}

The mass-energy density approach of field theory is equivalent to Einstein's me- 
tric-based theory of curved space-time. Although Einstein's metric-based general relativity is by far the most familiar theory of gravity, a number of approaches have formulated gravity as a gauge theory. In 1954, Gupta constructed a theory in which the "source" couples to the massless spin-2 field $h_{\mu \nu}$ as the energy-momentum tensor, including the energy momentum of the $h_{\mu v}$ field itself. The coupling induces a cubic term in the Lagrangian, resulting in a corresponding cubic term ${ }^{3} T^{\mu \nu}$ in the energy momentum tensor, which is then included in the source [22]. This in turn generates a quartic term ${ }^{4} T^{\mu v}$, and so on. This considers the stress-energy carried by the linearized gravitational field, $h_{\mu v}$ and iteratively corrects for it and then corrects the corrections. This alternate way to derive general relativity has been developed and explored by Gupta (1954), Kraichnan (1955), Thirring (1961), Feynman (1963) Weinberg (1965) and Deser (1970).

The most significant aspect of the gauge approach is that, per Feynman [23]: "this iterative procedure generates an infinite series that can be summed to yield the full nonlinear Einstein equation." Similarly, Misner, Thorne and Wheeler [24]: "Just as one can 'descend" from general relativity to linearized theory by linearizing about flat space time so can one 'bootstrap' one's way back up from linearized theory to general relativity...". The two formalisms are equivalent.

Although linear equations are transformable into non-linear via iterative analysis, the nonlinear equations did not just "snap into being". As Padmanabhan noted, it is necessary to know beforehand that the final field equations have to match with those in Einstein's theory, in order to introduce the extra assumptions to obtain it, but these extra assumptions are essentially equivalent to the result we're attempting to derive! So Einstein's equations did not just fall out of an analysis of gravity; or even the Equivalence Principle; many issues still have yet to be resolved. One simply cannot obtain an expression for the energy-momentum tensor for the spin-2 field that is unique and gauge invariant; instead one can obtain a large class of non-unique theories. This has resulted in numerous "Einstein-like" theories, a number of which are finally being eliminated by the real gravitational wave data that is increasingly being detected.

Ohanian and Ruffini observe that "almost all of the result that had been the subject of experimental investigation can be described by the linear approximation ... the deflection of light, the time delay of light, gravitational time dilation, gravitational lensing, and gravitational radiation emerge from the linear approximation." Recently Will [25] derived a new contribution to Mercury's perihelion advance, based in part on interaction between Mercury's motion and the gravitomagnetic field of moving planets; a contribution 100 times larger than the second-post-Newtonian contribution.

Poisson and Will [26] begin their development of post-Newtonian theory by postulating a form of the metric and ask "which guiding principle can be invoked to justify the choices made...? The answer is simply that no such principle exists..." The central theme of their book is "the physics of weak gravitational 
fields." A reason for this approach is that "no exact solution to Einstein's equation has ever been found that describes a simple double-star system and orbital motion." Thus the focus on weak field approximation is utilitarian of necessity, as they conclude that "Almost no physically useful exact solutions of the theory (of general relativity) are known." Yet "we have no good understanding of why this approximation to general relativity should be so effective," since "neutron stars... have very strong internal gravity".

In contrast, the Self-Interaction Principle makes no field strength assumption, other than the implicit assumption that the strength of the field at the big bang is included in the theory.

\section{Conclusions}

Despite equivalence of the linear formalism to Einstein's nonlinear form, the "weak field approximation" terminology has misled physicists to believe that real gravitation is described by space-time curvature corresponding to the nonlinear formalism, although Feynman, Padmanabhan, Weinberg, and others insist that curved spacetime is not a necessary conception of gravity.

Familiarity with the Schwarzschild and Kerr metrics convince many that the "proper" theory of gravity is general relativity. Nevertheless, these metric solutions are static; they represent a geometric solution that does not evolve over time, given a fixed mass, $M$. For a dynamic space-time, an exact metric solution to Einstein's field equations has existed for over 90 years, yet its interpretation has been "obscure and questionable". This Kasner metric has recently been interpreted [27] in terms of self-interaction equations, and a meaningful physical theory derived.

We have worked from the assumption that our universe evolved from a single primordial field. The corresponding Principle of Self-interaction produces the known gravitomagnetic field equations; however, in contrast with the "weak field approximation" assumption, a self-interacting field remains self-interacting-it does not become non-self-interacting due simply to a physicist's deleting nonlinear terms for ease of solution. Therefore the most significant aspect of the derivation of the gravitational field equations from the self-interaction principle is that there is absolutely no mention of field strength. Derivation from the Principle of Self-interaction instead yields all-strength-field-equations of the gravitational field.

The space-time curvature aspect of gravity is based on an approximate equivalence principle that holds only at a mathematical point. On the other hand, there is another assumed equivalence that some find remarkable; Ohanian and Ruffini state:

"That the exact nonlinear equations are implied by the linear equations... is a remarkable feature of Einstein's theory."

We restate this as follows: 
That Einstein's exact nonlinear equations are implied by our linear equations... is a remarkable feature of the Principle of Self-interaction.

This offers a new ontological understanding of physical reality.

\section{Conflicts of Interest}

The author declares no conflicts of interest regarding the publication of this paper.

\section{References}

[1] Einstein, A. (1952) Relativity: The Special and General Theory. Crown Publishers, New York

[2] Hertz, H. (1890) On the Fundamental Equations of Electromagnetics for Bodies in Motion. In: Electric Waves, Dover Publications, New York, 195-240.

[3] Heaviside, O. (1893) The Electrician, 31, 281-282.

[4] Abbott, B., et al. (2016) Physical Review Letters, 116, Article ID: 061102.

[5] Feynman, R. (1965) Lectures on Gravitation. Westview Press, Boulder.

[6] Susskind, L. (2006) The Cosmic Landscape. Little, Brown and Co., New York.

[7] Wilczek, F. (1998) The Future of Particle Physics as a Natural Science.

[8] Will, C. (2011) On the Unreasonable Effectiveness of the Post-Newtonian Approximation in Gravitational Physics.

[9] Yau, S.-T. (2010) The Shape of Inner Space. Basic Books, New York.

[10] Danforth, D. (2007) Private Communications.

[11] Wikipedia, Birkhoff Shell Theorem.

[12] Macdonald, A. (2012) Vector and Geometric Calculus. CreateSpace Pub., Charleston.

[13] Hestenes, D. and Sobcyzk, G. (1984) Clifford Algebra to Geometric Calculus. Reidel Pub. Co., Boston. https://doi.org/10.1007/978-94-009-6292-7

[14] Ohanion, H. and Ruffini, R. (2013) Gravitation and Spacetime. 3rd Edition, Cambridge U. Press, New York. https://doi.org/10.1017/CBO9781139003391

[15] Smith, J., et al. (2018) Physical Review Letters, 120, Article ID: 143002. https://doi.org/10.1103/PhysRevLett.120.143002

[16] Jefimenko, O.D. (2006) Gravitation and Cogravitation. Electret Scientific Pub., Star City.

[17] Everitt, C., et al. (2011) Gravity Probe B: Final Results.

[18] Abbott, B, et al. (2017) Physical Review Letters, 199, Article ID: 161101.

[19] Will, C. (2018) Theory and Experiments in Gravitational Physics. 2nd Edition, Cambridge University Press, Cambridge.

[20] Arthur, J. (2011) Understanding Geometric Algebra for Electromagnetic Theory. Wiley, Hoboken. https://doi.org/10.1002/9781118078549

[21] Kauffmann, S. (2014) Unique Einstein Gravity from Feynman's Lorenz Condition.

[22] Padmanabhan, T. (2010) Gravitation Foundation and Frontiers. Cambridge University Press, Cambridge. https://doi.org/10.1017/CBO9780511807787

[23] Feynmann, R. (1964) Feynman Lectures on Gravitation. Westview Press, Boulder.

[24] Meisner, C., Thorne, K. and Wheeler, J. (1971) Gravitation. W H Freeman Co., New 
York.

[25] Will, C. (2018) Physical Review Letters, 120, Article ID: 191101. https://doi.org/10.1103/PhysRevLett.120.191101

[26] Poisson, E. and Will, C. (2014) Gravity, Newtonian, Post-Newtonian, Relativistic. Cambridge University Press, Cambridge.

[27] Klingman, E. (2019) Prespacetime Journal, 10, 671-680. 